\title{
Trapped field properties of a Y-Ba-Cu-O bulk by pulsed field magnetization using a split coil inserted by iron yokes with various geometries and electromagnetic properties
}

K. Takahashi ${ }^{1)}$, M. D. Ainslie ${ }^{2)}$, H. Fujishiro ${ }^{1)}$, T. Naito ${ }^{1)}$, Y-H. Shi ${ }^{2)}$, and D. A. Cardwell ${ }^{2)}$

${ }^{1}$ Department of Physical Science and Materials Engineering, Faculty of Science and Engineering, Iwate University, 4-3-5 Ueda, Morioka 020-8551, Japan

2 Bulk Superconductivity Group, Department of Engineering, University of Cambridge, Trumpington Street, Cambridge CB2 1PZ, UK

\section{$\underline{\text { Abstract }}$}

We have investigated, both experimentally and numerically, the trapped field characteristics of a standard Y-Ba-Cu-O bulk of $30 \mathrm{~mm}$ in diameter and $14 \mathrm{~mm}$ in thickness magnetized by pulsed field magnetization (PFM) using a split coil, in which three kinds of iron yoke are inserted in the bore of the coil: soft iron with a flat surface, soft iron with a taper, and permendur (50Fe+50Co alloy) with a flat surface. The highest trapped field, $B_{\text {Tmax }}$, of $2.93 \mathrm{~T}$ was achieved at $40 \mathrm{~K}$ in the case of the permendur yoke, which was slightly higher than that obtained for the flat soft iron or the tapered soft iron yokes, and was much higher than $2.20 \mathrm{~T}$ in the case without the yoke. The insertion effect of the yoke on the trapped field characteristics was also investigated using numerical simulations. The results suggest that the saturation magnetic flux density, $B_{\text {sat }}$, of the yoke acts to reduce the flux flow due to its hysteretic magnetization curve and the higher electrical conductivity, $\sigma$, of the yoke material also acts to suppress the flux increase rate. A flux jump (or flux leap) can be reproduced in the ascending stage of PFM using numerical simulation, using an assumption of relatively high $J_{\mathrm{c}}(T$, $B$ ) characteristics. The insertion effect of the yoke with high $B_{\text {sat }}$ and $\sigma$ is discussed to enhance the final trapped field from both electromagnetic and thermal points of view.

\section{$\underline{\text { Keywords }}$}

Bulk superconductor; Pulsed field magnetization; Numerical simulation; Trapped field, Iron based yoke; Electromagnetic property

\section{Introduction}

$\mathrm{RE}-\mathrm{Ba}-\mathrm{Cu}-\mathrm{O}$ (RE: rare earth element or $\mathrm{Y}$ ) bulk superconductors have promising potential to be used as a strong trapped field magnet (TFM), which can trap a magnetic field of several Tesla 
because of their high critical current density, $J_{c}[1,2]$. This property can be exploited in practical applications, such as magnetic separation, magnetic drug delivery systems and sputtering cathodes for thin film growth [3-5]. The field cooling magnetization (FCM) technique, which needs a large and expensive experimental setup using a superconducting coil magnet, is commonly used for fundamental measurements on bulk superconductors to give the best indication of the capability of the sample as a TFM. A record-high trapped field of $17.6 \mathrm{~T}$ has been achieved in a stack of two Gd-Ba-Cu-O bulks at $26 \mathrm{~K}$ by FCM [6]. Pulsed field magnetization (PFM) has been also applied as a practical magnetizing method for high TFMs because of its inexpensive and mobile setup, unlike FCM. However, a significant challenge to enhance the trapped field by PFM is the reduction of large temperature rise caused by the dynamical magnetic flux motion, especially at low temperatures, in which the magnetic flux quickly moves in the bulk on the order of milliseconds [7-10]. Using a multi-pulse technique, named a modified multi-pulse technique with stepwise cooling (MMPSC), we successfully achieved a highest trapped field of $5.20 \mathrm{~T}$ on a $45 \mathrm{~mm}$ Gd-Ba-Cu-O disk bulk at $30 \mathrm{~K}$, which is a record-high trapped field by PFM to date [11].

In recent years, a ferromagnetic yoke has been inserted in the magnetizing fixture to increase the trapped magnetic field $[12,13]$, in which permendur $(50 \mathrm{Fe}+50 \mathrm{Co}$ alloy) and soft iron were used experimentally as a yoke material. We have also achieved a trapped field of over $3 \mathrm{~T}$ at $40 \mathrm{~K}$ on Gd-Ba-Cu-O disk bulk by PFM, employing a split coil with a pair of soft iron yokes [14], in which a symmetric trapped field profile can be also realized [15]. In addition, for the $\mathrm{MgB}_{2}$ bulk, we achieved over $1 \mathrm{~T}$ at $13 \mathrm{~K}$ by PFM without any flux jumps on a high- $J_{\mathrm{c}} \mathrm{MgB}_{2}$ disk bulk using the split coil with soft iron yoke, which is a record-high trapped field by PFM for the $\mathrm{MgB}_{2}$ bulk to date [16]. Numerical simulation is a powerful tool to consider the obtained experimental results during PFM, and to understand the flux dynamics using electromagnetic and thermal equations. Several studies using the numerical simulation have been reported for PFM [17-23]. We have previously confirmed the advantage of the insertion of a soft iron yoke with a flat surface in the split coil numerically, and compared this to the case without the yoke $[14,15]$.

However, there is no systematic investigation yet on the effects of varying the electromagnetic properties and the shape of the yokes on the trapped field characteristics, both experimentally and numerically, to enhance the trapped field by the PFM technique. In this paper, we investigate the trapped field characteristics of a Y-Ba-Cu-O bulk magnetized by PFM using a split coil, in which three kinds of iron yokes were inserted in the bore of the coil: a conventional soft iron with a flat surface, soft iron with a taper and a permendur with a flat surface. The effects of the material and the shape of yokes are discussed from electromagnetic and thermal points of view.

\section{Experimental Setup}

A typical Y-Ba-Cu-O bulk superconductor of $30 \mathrm{~mm}$ in diameter and $14 \mathrm{~mm}$ in thickness, which 
was fabricated by the top-seeded, melt-growth (TSMG) method in Cambridge [24], was mounted in a copper holder and was connected to the cold stage of a Gifford McMahon (GM) cycle helium refrigerator. A schematic view of apparatus is shown in Fig. 1(a). A split coil, which was cooled using liquid nitrogen, was placed outside the vacuum chamber, and the bulk disk was magnetized using the condenser bank $(60 \mathrm{mF})$ between the two coils of the split coil. Three kinds of iron yokes, as shown in Fig. 1(b), were inserted in the bores of the split coil: soft iron with a flat surface, soft iron with a taper and permendur with a flat surface. The each yoke were placed at a distance of 5 $\mathrm{mm}$ from the bulk top or bottom surface. Figure 2 presents the initial magnetization curve of the soft iron and permendur at $300 \mathrm{~K}$ measured by a SQUID magnetometer under an applied field, $B_{\text {app}}$, of up to $6 \mathrm{~T}$. The saturation magnetic flux density, $B_{\text {sat }}$, was determined to be $1.90 \mathrm{~T}$ for the soft iron and $2.13 \mathrm{~T}$ for the permendur at $B_{\mathrm{ex}}>1.0 \mathrm{~T}$, respectively. Pulsed applied fields, $B_{\mathrm{ex}}$, of amplitudes ranging from 2.24 to $5.67 \mathrm{~T}$ were applied individually to the bulk, cooled to an initial temperature of either $T_{\mathrm{s}}=65$ or $40 \mathrm{~K}$. The PFM experiments were performed for four cases of the yoke: (i) without any yoke, (ii) with soft iron, (iii) with tapered soft iron and (iv) with permendur. The time evolution of the local field, $B_{\mathrm{y}}(t)$, at the center of the bulk surface was measured during PFM by using the Hall sensor adhered on the center of the bulk and a digital oscilloscope. $B_{\mathrm{y}}(t)$ at $t=300 \mathrm{~ms}$ was referred to as a final trapped field, $B_{\mathrm{T}}$, after allowing sufficient time to relax the flux flow. The time evolution of the temperature, $T(t)$, was measured by a CERNOX ${ }^{\mathrm{TM}}$ thermometer on the side surface of the sample holder. After the magnetic pulse, the split coil was removed and the trapped field profile was mapped on the outer surface of the vacuum chamber, which is $2 \mathrm{~mm}$ above the bulk surface, by scanning an axial-type Hall sensor, with a pitch of $1 \mathrm{~mm}$, using an $x-y$ stage controller. The relationship between $B_{\mathrm{ex}}$ at the center of the bulk surface and the coil current flowing through a shunt resistor was calibrated for each yoke at $100 \mathrm{~K}$ above the transition temperature of the bulk. Figure 3 shows the time evolution of the local field, $B(t)$, normalized by those maxima, $B_{\text {ex }}$, measured at the center of the bulk surface at $100 \mathrm{~K}$ for the split coil with and without the several types of yokes. The rise time, $\tau$, at which the magnetic pulse takes a maximum value, was $18 \mathrm{~ms}$ for the case without any yoke, and $23 \mathrm{~ms}$ for three cases with a yoke. The presence of the yoke delayed the pulsed field during the ascending stage due to the induced eddy current and the descending stage due to its hysteretic magnetization. Note that the eddy current heating, which comes from the copper holder, was approximately $1.5 \mathrm{~K}$ when applying the pulsed field of $4 \mathrm{~T}$ at $100 \mathrm{~K}$ whether the yoke was present or not. 
(a)

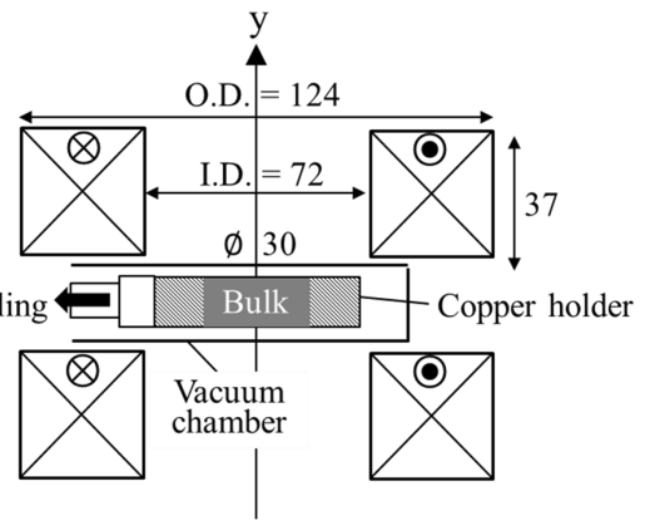

(b)

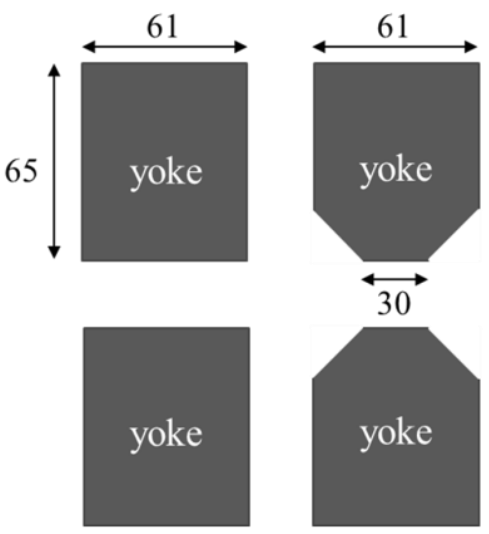

$(\mathrm{mm})$

Fig. 1. (a) Experimental setup around the disk bulk for PFM using a split coil. (b) The shape of the yoke pair with a flat surface for the soft iron and the permendur (left) and with a taper for the soft iron (right). The dimensions of the magnetizing coil and two types of yoke are also shown.

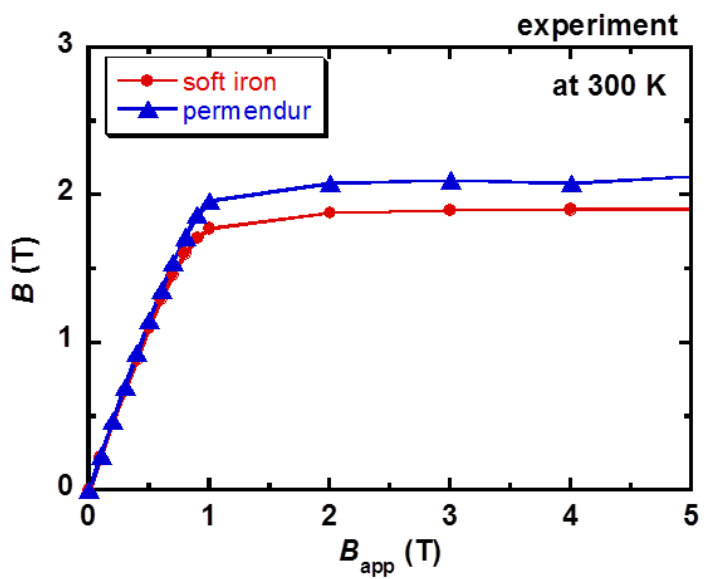

Fig. 2. Initial magnetization curves of the soft iron and the permendur at $300 \mathrm{~K}$ under an applied magnetic field, $B_{\text {app. }}$.

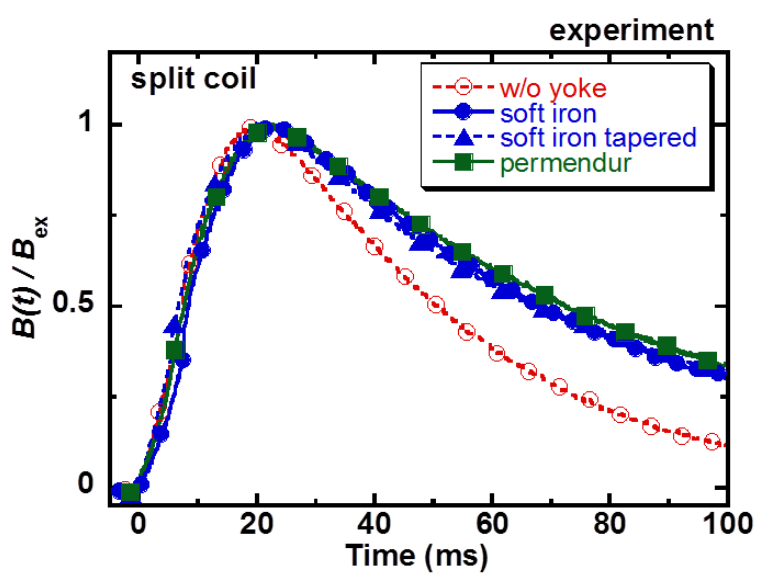

Fig. 3. Time evolution of the local field, $B(t)$, normalized by those maxima, $B_{\text {ex }}$, measured at the center of the bulk surface at $100 \mathrm{~K}$ for the split coil with and without the several types of yokes. The ramp and decay rates of the magnetic pulse were delayed by the yoke insertion. 


\section{Experimental Results}

In this section, we present the typical behavior of the magnetic flux and the influence of the magnetic properties and geometries of the yoke on the trapped field characteristics. Figure 4 presents the experimental results for the final trapped field, $B_{\mathrm{T}}$, at the center of the bulk surface, as a function of the applied field, $B_{\text {ex }}$, using the split coil without a yoke and with the three types of yokes at $65 \mathrm{~K}$ and $40 \mathrm{~K}$. A critical applied pulsed field is necessary for the full penetration of the magnetic flux into the bulk center. At $65 \mathrm{~K}$, as shown in Fig. 4(a), $B_{\mathrm{T}}$ started to increase at $B_{\mathrm{ex}}=2.5$ $\sim 3.0 \mathrm{~T}$ and abruptly increased with increasing $B_{\mathrm{ex}}$ in all cases. A characteristic behavior, which is so-called flux jumps (or leaps), has been reported for a high- $J_{c}$ bulk sample during PFM [25, 26], where the magnetic flux suddenly intruded towards the center of the bulk. We abbreviate the optimum applied field as $B_{\mathrm{ex}}{ }^{*}$, at which the bulk achieved the maximum trapped field, $B_{\text {Tmax }}$. Higher $B_{\text {Tmax }}$ and higher $B_{\mathrm{ex}}{ }^{*}$ were obtained for the cases with a yoke, compared to without any yoke. Obvious differences appeared more obviously at $40 \mathrm{~K}$ as shown in Fig. 4(b) due to the difference in the magnetic flux motion and temperature rise between these four cases. The highest trapped field of $B_{\text {Tmax }}=2.93 \mathrm{~T}$ was achieved for the permendur yoke at $40 \mathrm{~K}$, which was higher than that without any yoke and any other cases (flat or tapered soft iron). Note that the maximum trapped field decreased to $2.73 \mathrm{~T}$ after removing the split coil with the yoke for the measurement of the trapped field profile. There were no significant differences in the trapped field based on the magnetic properties of the yoke material between the soft iron and permendur. The $B_{\text {Tmax }}$ value for the tapered soft iron yoke was smaller than that for the soft iron yoke with a flat surface. Although the tapered soft iron yoke was expected to concentrate the magnetic flux towards the center of the sample, there is no significant effect to enhance the trapped field. 

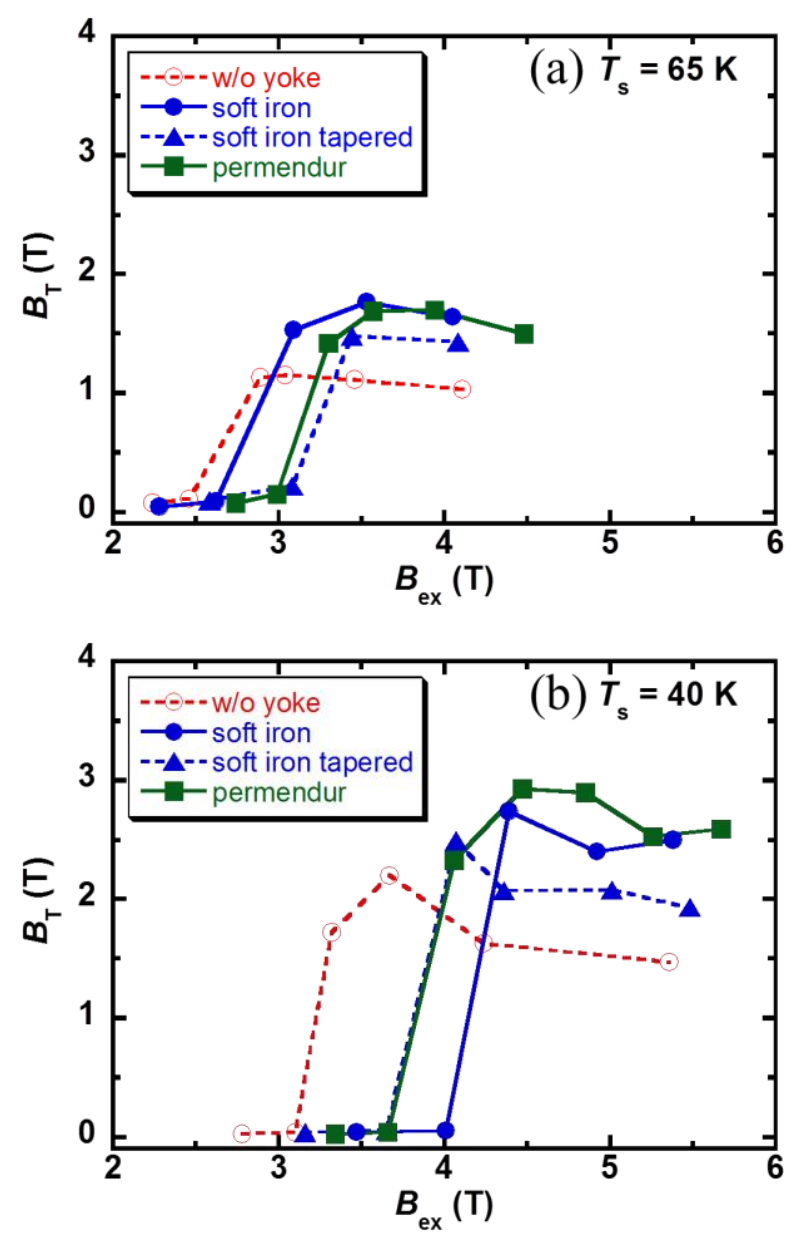

Fig. 4. Experimental results of the final trapped field, $B_{\mathrm{T}}$, at the center of the bulk surface, as a function of the applied field, $B_{\mathrm{ex}}$, using the split coil without any yoke and with the three types of yokes at (a) $65 \mathrm{~K}$ and (b) $40 \mathrm{~K}$.

Figure 5 shows the trapped field profiles, $B_{\mathrm{T}}(2 \mathrm{~mm})$, measured at $2 \mathrm{~mm}$ above the bulk surface at $40 \mathrm{~K}$ before full magnetization under applying the pulsed field of $B_{\text {ex }}<4.0 \mathrm{~T}$, and after full magnetization under applying the pulsed field of $B_{\mathrm{ex}}>4.2 \mathrm{~T}$ using the split coil (a) without any yoke and (b) with soft iron of flat surface, (c) with tapered soft iron and (d) with permendur of flat surface. Before the full magnetization for all cases of (a1) - (d1), the trapped field profiles were of a convex shape, showing that the magnetic flux was mainly trapped at the grain sector boundaries (GSBs) due to the inhomogeneous $J_{\mathrm{c}}$ distribution in the bulk, typically observed with TSMG-grown, bulk Y-Ba-Cu-O samples [7]. On the other hand, for the cases of (a2) - (d2) at $B_{\text {ex }}>4.2 \mathrm{~T}$, the trapped field profiles exhibited a conical shape. The difference in the trapped field between the cases without any yoke (a2) and with a yoke (b2 - d2) remained significant, even at $2 \mathrm{~mm}$ above the bulk surface, although the magnitude of magnetic field is severely decreased and broadened with increasing distance from the bulk surface. The magnetic flux density in the case of the soft iron (b2) and permendur (d2) was twice as high as that obtained in the case without any yoke (a2). It is clear that a higher trapped field with a conical profile can be obtained in the cases with a flat yoke (b2 
and d2), compared to the tapered soft iron (c2).

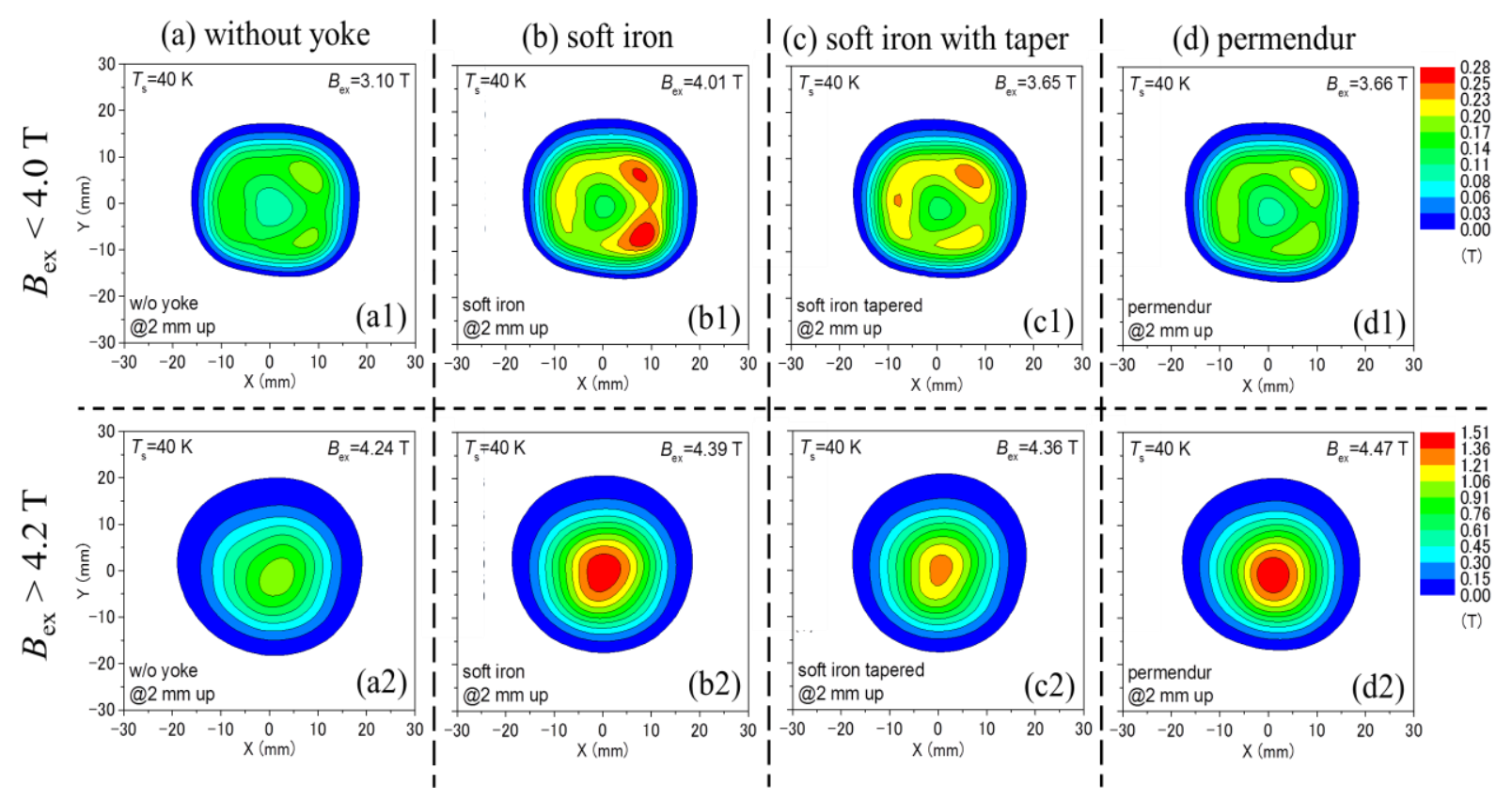

Fig. 5. Trapped field profiles, $B_{\mathrm{T}}(2 \mathrm{~mm})$ measured at $2 \mathrm{~mm}$ above the bulk surface at $T_{\mathrm{s}}=40 \mathrm{~K}$ below full magnetization after applying $B_{\mathrm{ex}}<4.0 \mathrm{~T}$ (upper panels), using the split coil without any yoke (a1) and with three types of yoke: soft iron with a flat surface (b1), soft iron with a taper (c1) and permendur with a flat surface (d1). The results above full magnetization, after applying $B_{\mathrm{ex}}>4.2 \mathrm{~T}$ using the split coil without any yoke (a2) and with three types of yoke: soft iron (b2), tapered soft iron (c2) and permendur (d2), are also shown in the lower panels.

Figure 6 shows the time dependence of the local field, $B_{\mathrm{y}}(t)$, at the center of the bulk surface after applying the pulsed field of $B_{\mathrm{ex}} \cong 4.0 \mathrm{~T}$ at $65 \mathrm{~K}$ (upper panels), and $B_{\mathrm{ex}} \cong 5.4 \mathrm{~T}$ at $40 \mathrm{~K}$ (bottom panels), using the split coil (a) without any yoke and with three types of yoke: (b) soft iron with a flat surface, (c) tapered soft iron and (d) permendur with a flat surface. The applied pulsed field, $B_{\mathrm{ex}}(t)$, is also shown in each figure. For the case without any yoke at $65 \mathrm{~K}$, as shown in (a1), the magnetic flux did not penetrate early in the ascending stage of PFM due to the shielding effect of the bulk, and then abruptly starts to intrude into the bulk, where a flux jump occurred at $t=10$ ms. The magnetic flux behaved in the same way, regardless of whether the yoke was present or not. The flux jump was observed for all the cases, at $65 \mathrm{~K}$ and $40 \mathrm{~K}$ in the ascending stage of the pulse. Another effect of the yoke insertion can be also seen in the descending stage. The downward arrow shown in Fig. 6 presents the flux flow caused by the dynamic movement of the magnetic flux associated with the Lorentz force and the temperature rise. For the case without any yoke at $65 \mathrm{~K}$ (a1), the magnetic field decreased significantly with increasing time. However, for the cases with a yoke ((b1) - (d1)) at $65 \mathrm{~K}$, the magnetic field still remained and was trapped with minimal flux flow, even after the whole process of magnetization $(t>300 \mathrm{~ms})$ [15]. The contribution of the yoke insertion during PFM exists mainly in the descending stage. Moreover, for the cases with a yoke 
$((\mathrm{b} 2)-(\mathrm{d} 2))$ at $40 \mathrm{~K}$, the maximum $B_{\mathrm{y}}(t)$ value was fairly smaller than the maximum $B_{\mathrm{ex}}(t)$, which is contrast to the cases at $65 \mathrm{~K}$. These results suggest that there is still some room left for further enhancement of the trapped field.

(a) without yoke
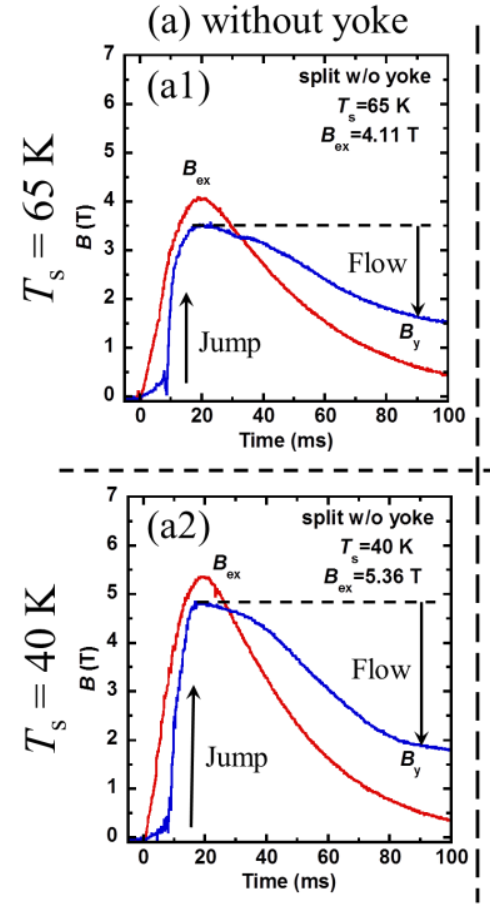

(b) soft iron

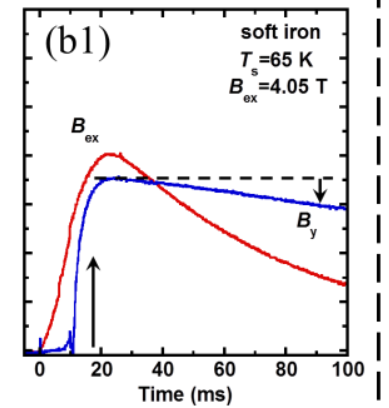

(c) soft iron with taper

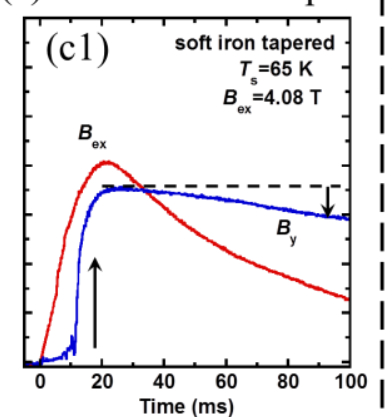

(d) permendur

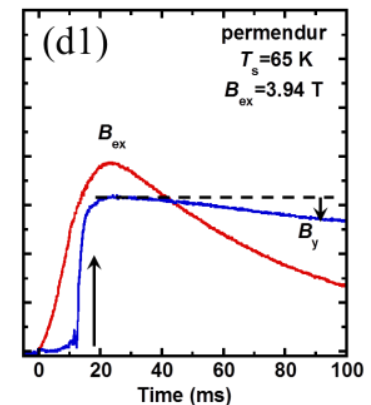

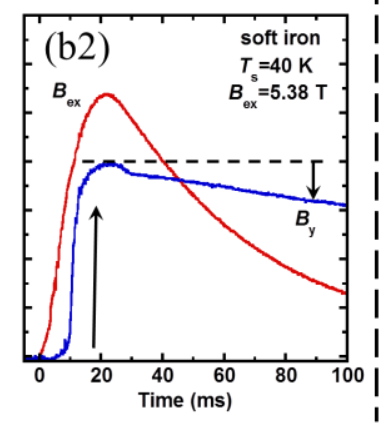
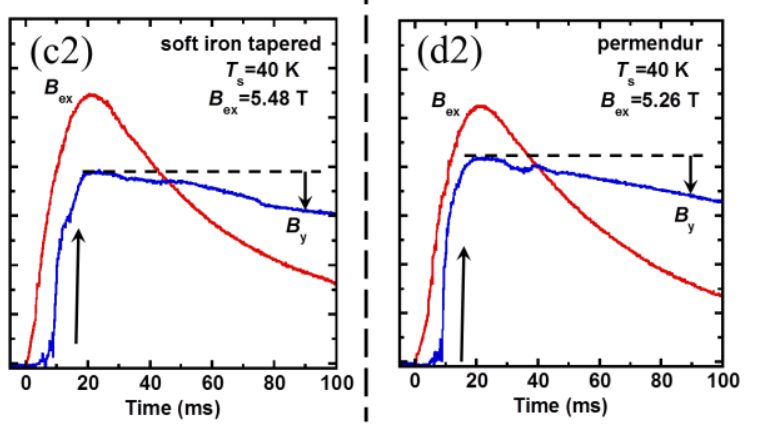

Fig. 6. Time dependence of the field, $B_{\mathrm{y}}(t)$, at the center of the bulk surface after applying the pulsed field of $B_{\text {ex }} \cong 4.0 \mathrm{~T}$ at $65 \mathrm{~K}$ (upper panels), and $B_{\text {ex }} \cong 5.4 \mathrm{~T}$ at $40 \mathrm{~K}$ (lower panels), using the split coil (a) without any yoke and with three types of yoke: (b) flat soft iron, (c) tapered soft iron and (d) flat permendur.

Considering the flux increase rate is effective to compare the magnetic flux behavior [28]. Figure 7 shows the time dependence of the flux increase rate, $R_{\mathrm{F}}$, after applying $B_{\mathrm{ex}} \cong 4.0 \mathrm{~T}$ at $65 \mathrm{~K}$, which is defined by $\mathrm{d} B_{\mathrm{y}}(t) / \mathrm{d} t$ in Figs. 6(a1) $\sim(\mathrm{d} 1)$. The $R_{\mathrm{F}}$ value abruptly increased and took a maximum value at the same time as the occurrence of the flux jump during the ascending stage. Generally, the maximum $R_{\mathrm{F}}$ value increases with the increase in $B_{\mathrm{ex}}$ because a larger amount of magnetic flux must intrude into the bulk within a finite time with a constant ramp rate of the pulse. The maximum $R_{\mathrm{F}}$ values for any cases with a yoke were reduced, and these maxima were also delayed, compared to those of the case without any yoke, showing that the yoke acted to suppress the dynamical motion of the magnetic flux. The $R_{\mathrm{Fmax}}$ value for the case without any yoke is larger than that with a yoke because there is no disturbance of flux motion from the yoke.

The heat generation during PFM is caused by the pinning loss and viscous flow loss due to the dynamical magnetic flux motion [29]. Figure 8 shows the maximum temperature rise, $\Delta T$, measured on the side of the sample holder, as a function of the applied field, $B_{\mathrm{ex}}$, for the cases with and without a yoke at $65 \mathrm{~K}$ and $40 \mathrm{~K} . \Delta T$ increased monotonically with increasing $B_{\text {ex }}$, which is in contrast to the final trapped field shown in Fig. 4. The $\Delta T$ behavior is related with the $R_{\mathrm{F}}$ behavior 
shown in Fig. 7; the $\Delta T$ and $R_{\mathrm{F}}$ values in the case without any yoke are larger than those in the cases with a yoke. In the cases of the yoke insertion, the $\Delta T$ values for the tapered soft iron yoke are smaller than those for the permendur at higher $B_{\text {ex }}$. This reduction of the heat generation in the case of the soft iron yoke also contributes to the enhancement of the trapped field.

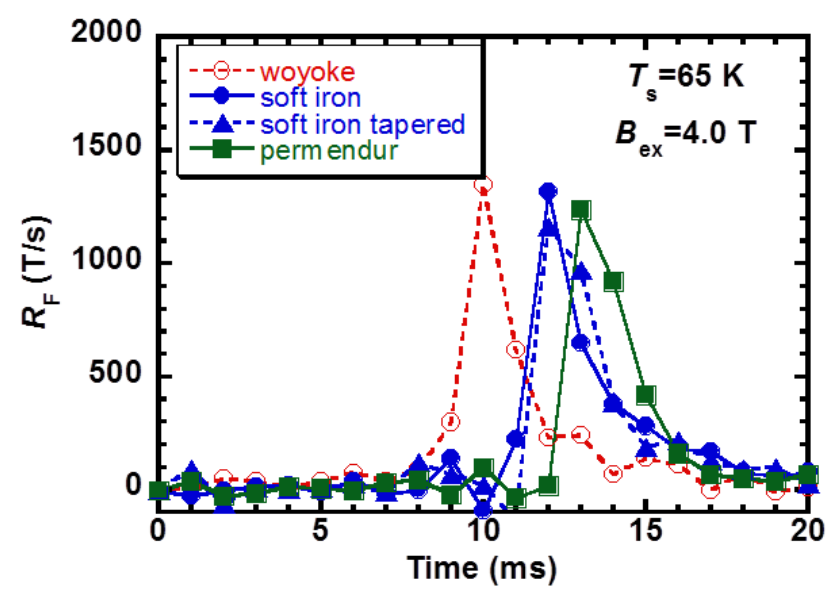

Fig. 7. Time dependence of the flux increase rate, $R_{\mathrm{F}}$, at the center of the bulk surface after applying $B_{\text {ex }} \cong 4.0 \mathrm{~T}$ at $65 \mathrm{~K}$ for all cases: with and without the three types of yoke. $R_{\mathrm{F}}$ was calculated from $\mathrm{d} B_{\mathrm{y}}(t) / \mathrm{d} t$ in Figs. $6(\mathrm{a} 1) \sim(\mathrm{d} 1)$.

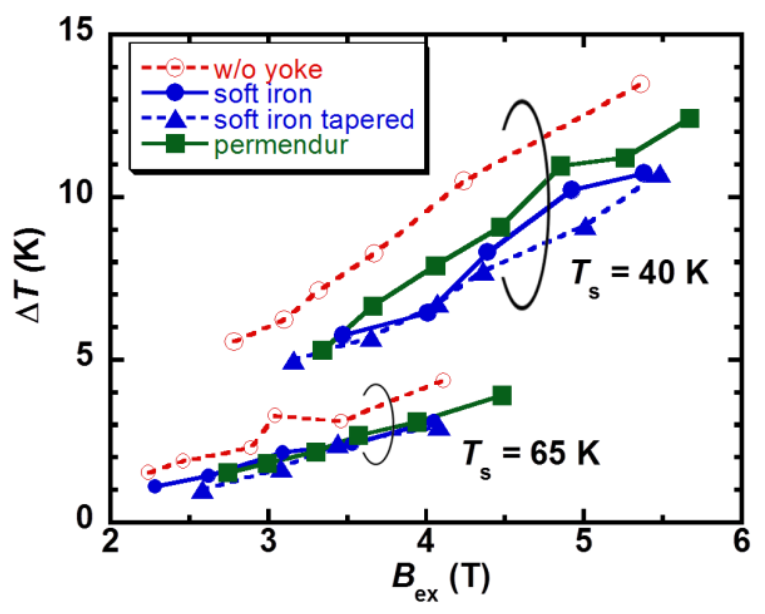

Fig. 8. Maximum temperature rise, $\Delta T$, measured on the side of the sample holder, as a function of the applied field, $B_{\mathrm{ex}}$, using the split coil without any yoke and with the three types of yoke at $65 \mathrm{~K}$ and $40 \mathrm{~K}$.

\section{Numerical Modelling}

In this section, the yoke insertion effect was considered in detail using numerical simulations, for which the typical behavior of the magnetic flux dynamics, such as a flux jump in the ascending stage, is reproduced, and a ferromagnetic yoke is confirmed to have a positive effect on the enhancement of the trapped field. Based on our experimental setup for PFM, as shown in Fig. 1, we constructed two-dimensional models using the finite element method (FEM) with a $1.0 \mathrm{~mm}$ mesh in size in the bulk. A schematic view of the modelling geometry is shown in Fig. 9 for (a) without any 
yoke, (b) soft iron and permendur with a flat surface and (c) soft iron with a taper. The physical phenomena during PFM are described by electromagnetic and thermal equations, which were referred from refs. [30, 31]. Commercial software, Photo-Eddy, combined with Photo-Thermo (Photon Ltd, Japan), was adopted for the analysis, which was carried out using a standard personal computer. The simulation procedure and the parameters used were described elsewhere in detail [32]. A modified power- $n$ law $(n=20)$, described in refs. [33, 34], was used to represent the non-linear $E-J$ characteristic of the bulk. The temperature and magnetic field dependence of critical current density, $J_{\mathrm{c}}(T, B)$, was described as

$$
J_{c}(T, B)=\alpha\left\{1-\left(\frac{T}{T_{c}}\right)^{2}\right\}^{\frac{3}{2}} \frac{B_{0}}{|B|+B_{0}},
$$

where $T_{\mathrm{c}}(=92 \mathrm{~K})$ is the critical temperature of the superconducting bulk. The bulk sample with the same dimensions as those used in the experiment was set to the sample holder and was cooled from the periphery to either $T_{\mathrm{s}}=65 \mathrm{~K}$ or $40 \mathrm{~K}$ before applying the pulsed field. The $J_{\mathrm{c}}(T, B)$ characteristics used in the simulation are shown in Fig. 10. In this simulation, the magnetic field dependence of $J_{\mathrm{c}}$ was described using the Kim model with $B_{0}=0.04$, and a constant $\alpha$ value of 1.5 $\mathrm{x} 10^{10} \mathrm{~A} / \mathrm{m}^{2}$, which is higher than that of an ordinary $\mathrm{Y}-\mathrm{Ba}-\mathrm{Cu}-\mathrm{O}$ bulk, was supposed in order to reproduce the flux jump in the ascending stage of the PFM process. Note that we have previously reproduced the flux jumps during PFM using numerical simulations, in which the $J_{\mathrm{c}}(T, B)$ was input directly using the experimentally measured $J_{\mathrm{c}}(T, B)$ characteristics of a small sample and the interpolation method [35]. In the thermal model, the temperature-independent anisotropic thermal conductivities $\kappa_{\mathrm{ab}}=20 \mathrm{Wm}^{-1} \mathrm{~K}^{-1}$ in the $a b$-plane and $\kappa_{\mathrm{c}}=4 \mathrm{Wm}^{-1} \mathrm{~K}^{-1}$ along the $c$-axis were assumed for the bulk.

The electromagnetic properties of the yokes were represented by a saturation magnetic flux density, $B_{\text {sat }}$, and an electrical conductivity, $\sigma$. $B_{\text {sat }}$ for the two yoke materials (soft iron and permendur) was input using the magnetization curves shown in Fig. 2. A constant $\sigma$ value of $2.5 \mathrm{x}$ $10^{8} \mathrm{~S} / \mathrm{m}$ was assumed to match the ramp rate of the experimentally applied magnetic pulse. The time dependence of the applied field, $B_{\mathrm{ex}}(t)$, was approximated as

$$
B_{e x}(t)=B_{e x} \frac{t}{\tau} \exp \left(1-\frac{t}{\tau}\right)
$$

where the pulse rise time, $\tau$, is $18 \mathrm{~ms}$. Figure 11 shows the results of the simulation of the time evolution of the normalized applied fields at the center of the bulk surface at $T=100 \mathrm{~K}$ for the split coil with and without the different types of yokes. In the figure, the dashed line shows the case with a yoke by a paramagnetic metal such as aluminum or copper, instead of soft iron (or permendur), in which a small delay in $B_{\text {ex }}(t)$ can be seen, compared to the case without any yoke due to the induced eddy currents. Hence, $\sigma$ acts to delay the rise time of the pulse because the yoke suppresses the 
magnetic field by induced eddy currents within it. For the cases of the soft iron yokes and permendur yoke, $B_{\text {sat }}$ largely acted to delay the sweep of the descending stage of the pulse due to its hysteresis magnetization. These results for the magnetic pulse obtained by simulation show a good agreement with the experimental results in Fig. 3.

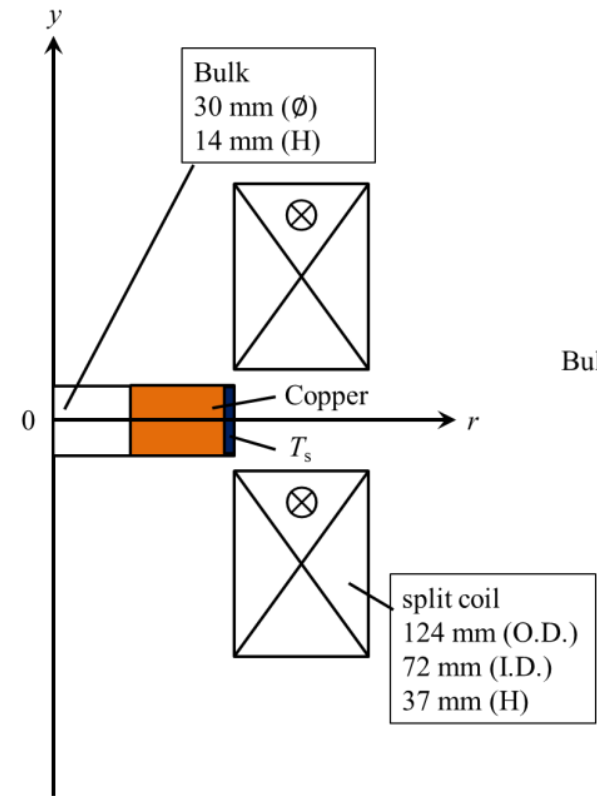

(a) w/o yoke

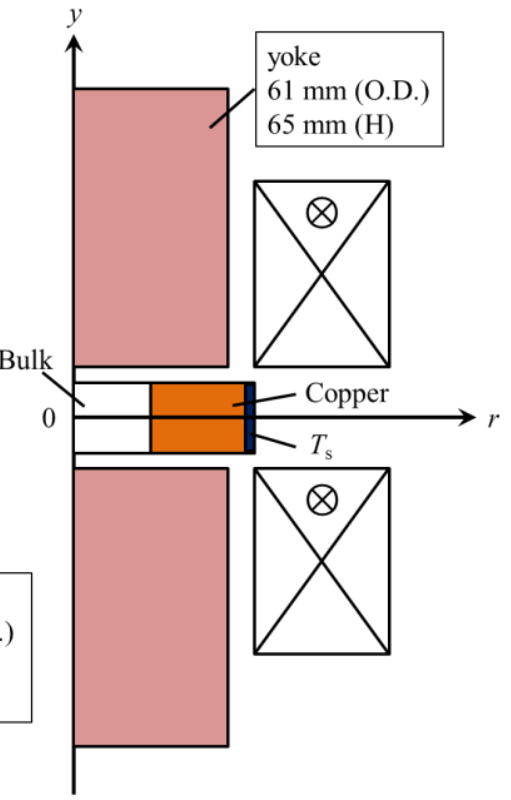

(b) soft iron, permendur

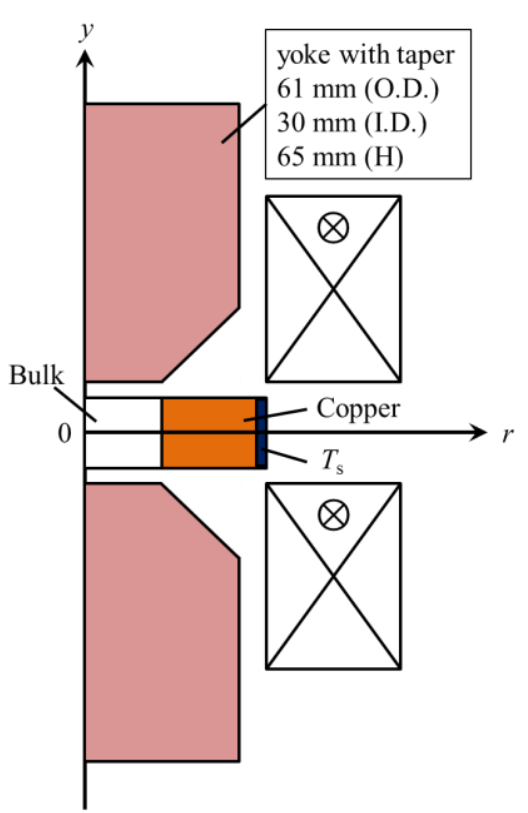

(c) soft iron with taper

Fig. 9. Schematic view of the modelling framework for PFM using a split coil, (a) without any yoke, (b) with soft iron and permendur with a flat surface and (c) with soft iron with a taper. The dimensions of the magnetizing fixture and the bulk are the same as the experimental setup shown in Fig. 1.

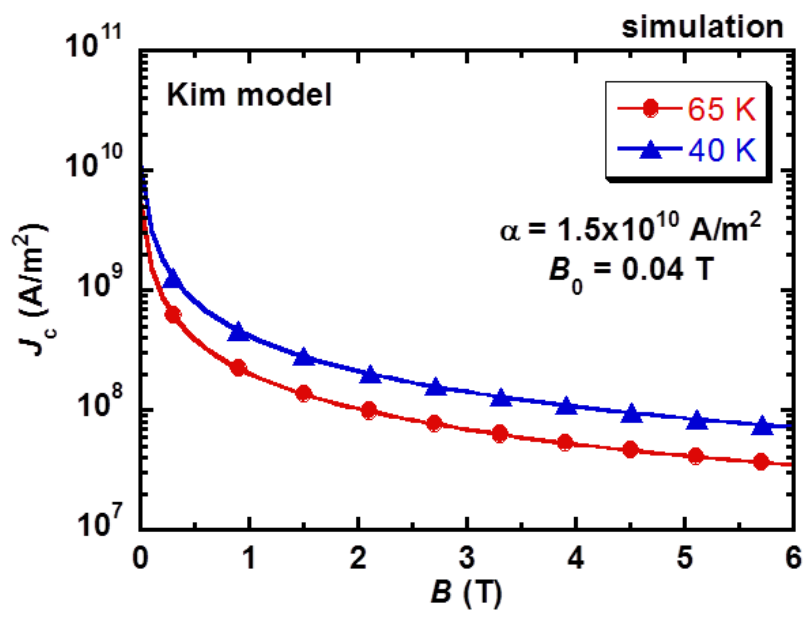

Fig. 10. The magnetic field dependence of critical current density, $J_{\mathrm{c}}$, at $65 \mathrm{~K}$ and $40 \mathrm{~K}$, represented by a Kim model. 


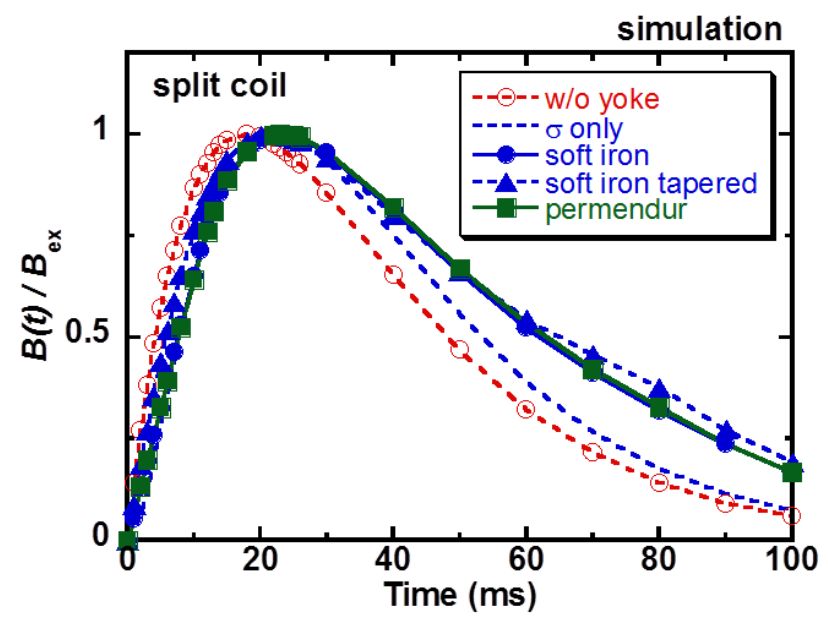

Fig. 11. Time evolution of the local field, $B(t)$, normalized by those maxima, $B_{\mathrm{ex}}$, at the center of the bulk surface for the split coil with and without the different yokes.

\section{Results of Numerical Simulation}

Similar results to those presented in the experimental results are shown in this section. Figure 12 shows the results of the numerical simulation of the final trapped field, $B_{\mathrm{T}}$, at the center of the bulk surface, as a function of the applied field, $B_{\mathrm{ex}}$, using the split coil without and with the three types of yoke at $65 \mathrm{~K}$ and $40 \mathrm{~K}$. The maximum trapped field, $B_{\text {Tmax }}$, for the cases with yoke was improved by $0.6 \mathrm{~T}$ at $65 \mathrm{~K}$ and $0.8 \mathrm{~T}$ at $40 \mathrm{~K}$, respectively, compared to the case without any yoke. However, there were no significant differences in the $B_{\mathrm{Tmax}}$ and $B_{\mathrm{ex}}{ }^{*}$ for the different yoke materials (soft iron or permendur) and different geometries (flat or taper). At $65 \mathrm{~K}, B_{\mathrm{T}}$ started to increase after applying $B_{\text {ex }}=2.5 \sim 3.0 \mathrm{~T}$, and drastically increased with only a slight increase in $B_{\mathrm{ex}}$, relating to the flux jumps observed in the case of without yoke. The results of the simulation show a good agreement with the experimental results, particularly at $65 \mathrm{~K}$ (shown in Fig. 4). At $T_{\mathrm{s}}=40 \mathrm{~K}$, the $B_{\mathrm{T}}$ vs. $B_{\text {ex }}$ curves are independent of the three types of yoke, but are larger than that for the case without any yoke. These results roughly reproduce the experimental ones in Fig. 4 , in which the $B_{\mathrm{T}}$ vs. $B_{\mathrm{ex}}$ curves slightly change depending on the three types yokes from the experiments in Fig. 2. The difference may result from that the estimated $J_{\mathrm{c}}(B)$ characteristics are different with those of realistic ones, as well as ignoring any inhomogeneity in $J_{c}$ around the ab-plane, which necessitates a 3D model [7].

\section{A: Magnetic flux behavior}

The results of the numerical analysis enable more detailed considerations about the magnetic flux behavior inside the bulk. First, the magnetic flux dynamics during PFM are presented for the case without any yoke. Figure 13 shows the time dependence of the local field, $B_{\mathrm{y}}(t)$, on the top surface of the bulk at positions $r=0 \mathrm{~mm}$ (center), $7 \mathrm{~mm}$ (middle) and $14 \mathrm{~mm}$ (edge) from the bulk 
center, after applying $B_{\mathrm{ex}}=4-5 \mathrm{~T}$ using the split coil without any yoke at $40 \mathrm{~K}$. The local field, $B_{\mathrm{y}}(t)$, at $r=14 \mathrm{~mm}$ near the bulk periphery essentially follows the applied field, $B_{\mathrm{ex}}(t)$, indicating that the magnetic flux intrudes into the bulk from its outside. Before full magnetization for $B_{\text {ex }}$ of (a) $4.00 \mathrm{~T}$ and (b) $4.30 \mathrm{~T}$, both of which were smaller than $B_{\mathrm{ex}}{ }^{*}=5 \mathrm{~T}$, a flux jump occurred from the edge to the middle position, but the amplitude of $B_{\text {ex }}$ and the corresponding flux jump was not enough for full penetration. The flux jump assisted the magnetic flux intrusion towards the center of the bulk with slightly increasing $B_{\mathrm{ex}}$ after full penetration, e.g., $B_{\mathrm{ex}}=4.50 \mathrm{~T}$, as shown in (c). This typical behavior of flux jumps or leaps, agrees well with our previous work using high $J_{\mathrm{c}}(T, B)$ characteristics based on experimental results [35].

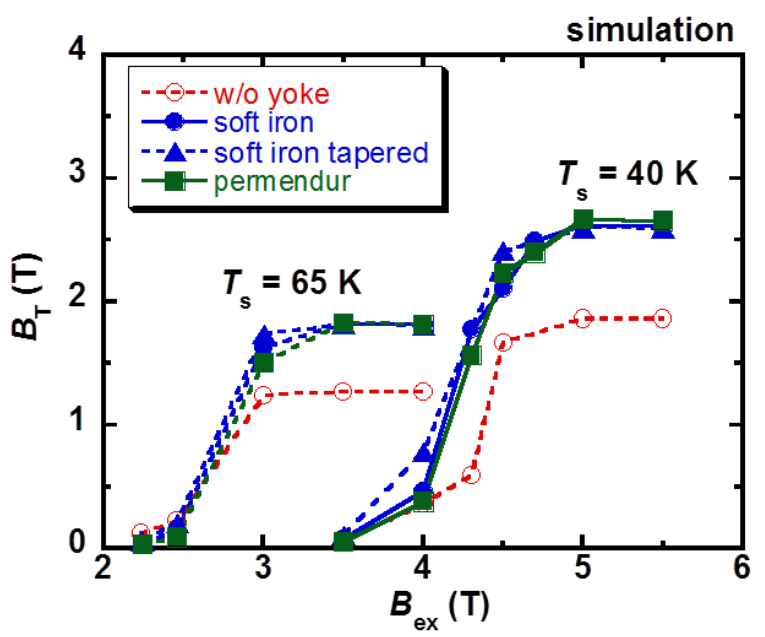

Fig. 12. Numerical simulation results of the final trapped field, $B_{\mathrm{T}}$, at the center of the bulk surface, as a function of the applied field, $B_{\text {ex }}$, using the split coil without any yoke and with the three types of yoke at $T_{\mathrm{s}}=65 \mathrm{~K}$ and $40 \mathrm{~K}$.
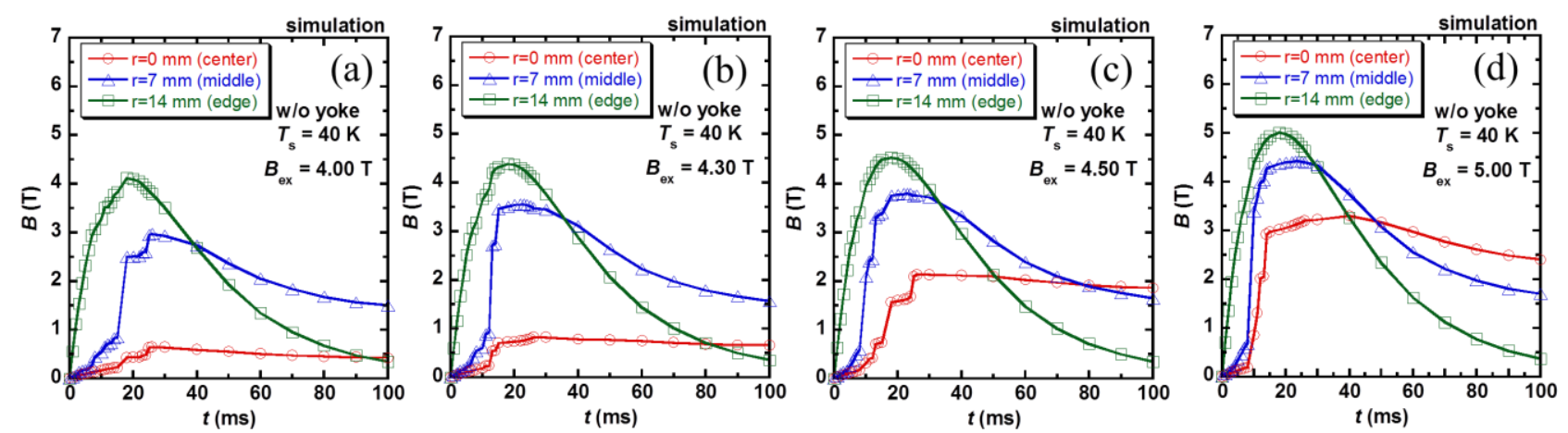

Fig. 13. Time dependence of the local fields, $B_{\mathrm{y}}(t)$, on the top surface of the bulk at positions of $r=$ $0 \mathrm{~mm}$ (center), $7 \mathrm{~mm}$ (middle) and $14 \mathrm{~mm}$ (edge) from the bulk center, after applying $B_{\mathrm{ex}}$ of (a) $4.00 \mathrm{~T}$, (b) $4.30 \mathrm{~T}$, (c) $4.50 \mathrm{~T}$ and (d) $5.00 \mathrm{~T}$ using the split coil without any yoke at $40 \mathrm{~K}$. Flux jumps assist the magnetic flux intrusion towards the center of the bulk with increasing $B_{\text {ex }}$. 
Figure 14 shows typical examples of the cross-sections of the trapped field profile in the bulk when a flux jump was confirmed at each point, after applying $B_{\mathrm{ex}}=5.0 \mathrm{~T}$ at $40 \mathrm{~K}$ using the split coil without any yoke, as shown in Fig. 14(d). The enlarged figure of Fig. 14(d) is shown again, in which the magnetic flux jumped twice at the middle and the center of the bulk. The bulk superconductor hinders the intrusion of the magnetic flux due to the shielding current, flowing within its capability of $J_{\mathrm{c}}$ below $T_{\mathrm{c}}$. Hence, the magnetic flux suddenly intruded into the bulk and a first flux jump occurred between $t=8 \mathrm{~ms}$ and $10 \mathrm{~ms}$. At $t=8 \mathrm{~ms}$, the gradient of magnetic flux became steep around $r=14 \mathrm{~mm}$ and then flux was intrudes into the bulk towards the middle of the bulk, overcoming the strong pinning force. The second small flux jump occurred between $13 \mathrm{~ms}$ and $14 \mathrm{~ms}$ near the center, at which the similar flux concentration can be seen around $r=7 \mathrm{~mm}$ and then was relaxed. In this way, the behavior of the magnetic flux related to the flux jump can be also confirmed in the cross section of the bulk using the numerical simulation. We are continuing to research the flux jump phenomenon during PFM, which requires some careful consideration due to the instabilities in the numerical model during such jumps. The jumps are related to the specific heat, and rate of change of $J_{\mathrm{c}}$ with respect to temperature (which can rise rapidly as the pulse is increasing) and/or magnetic field (depending on whether or not a Bean, Kim or other model is used). Qualitatively the model reproduces the flux jumps we observe experimentally, but improvements can and will be made quantitatively in the future.
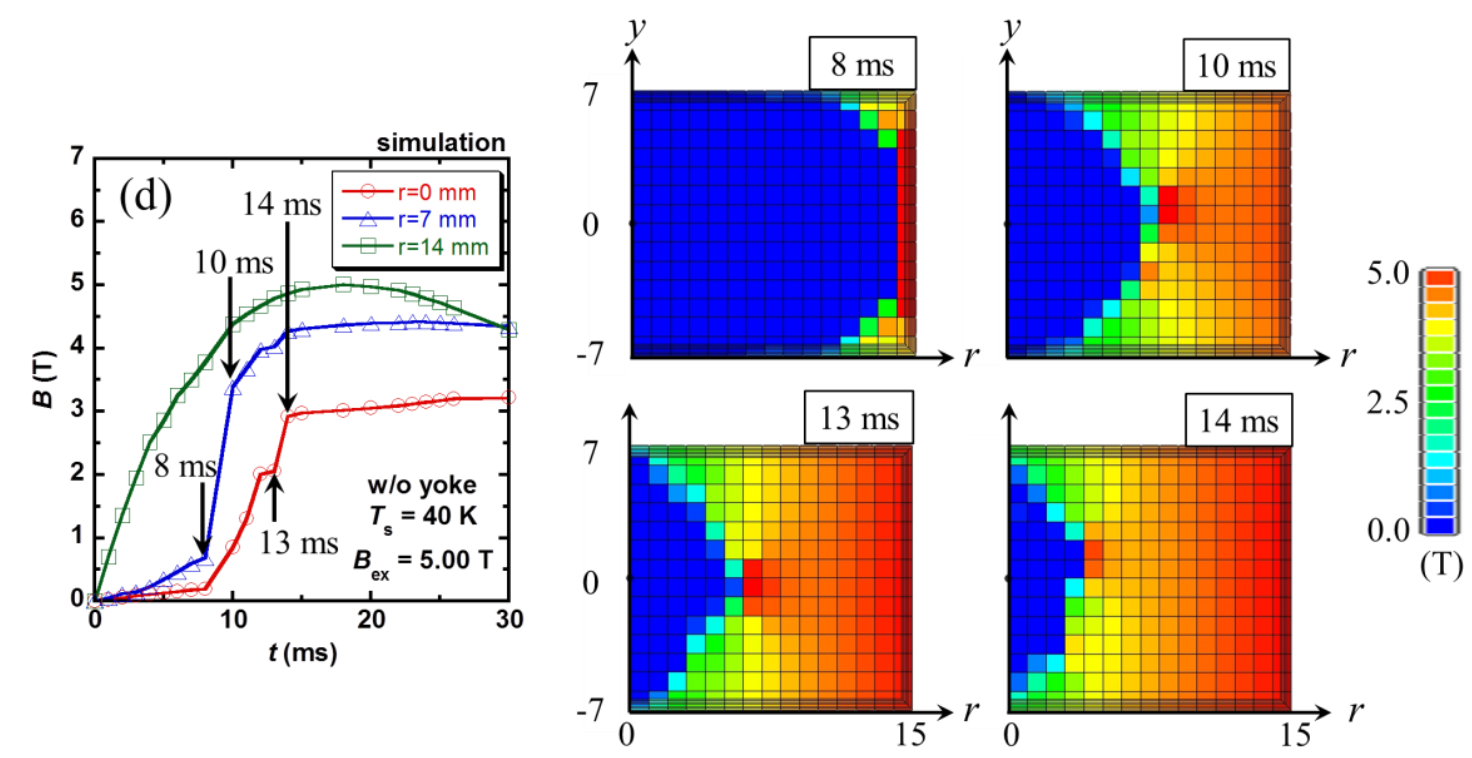

Fig. 14. Cross-section of the trapped field profile in the bulk, when flux jumps occurred at a time of $t=8,10,13$ and $14 \mathrm{~ms}$, after applying $B_{\mathrm{ex}}=5.0 \mathrm{~T}$ at $40 \mathrm{~K}$ using the split coil without any yoke (Right panels). The enlarged figure of Fig. 13(d) is shown in the left panel again.

\section{B: Yoke insertion effect}

Figure 15 shows the time dependence of the local field on the top surface of the bulk at each 
position of $r=0 \mathrm{~mm}$ (center), $7 \mathrm{~mm}$ (middle) and $14 \mathrm{~mm}$ (edge) from the bulk center, after applying the same applied field, $B_{\mathrm{ex}}=3.50 \mathrm{~T}$ at $65 \mathrm{~K}$ (upper panels) and $B_{\mathrm{ex}}=5.50 \mathrm{~T}$ at $40 \mathrm{~K}$ (lower panels), using the split coil (a) without any yoke and with the three types of yoke: (b) soft iron, (c) soft iron with a taper and (d) permendur. The magnetic flux suddenly intruded into the bulk, where a flux jump occurs in the bulk in the ascending stage at around $10 \mathrm{~ms}$ in all cases (a1) - (d1) at $65 \mathrm{~K}$ and (a2) - (d2) at $40 \mathrm{~K}$. For the cases without any yoke shown in (a1) and (a2), the magnetic flux decreased with increasing time in the descending stage. However, for the cases with a yoke (b1) (d1) or (b2) - (d2), the magnetic flux still remained trapped with minimal flux flow, even after the whole process of magnetization, in which the ferromagnetic yoke acts to reduce the flux flow. This behavior of the magnetic flux qualitatively agrees with the experimental results shown in Fig. 6.

(a) without yoke
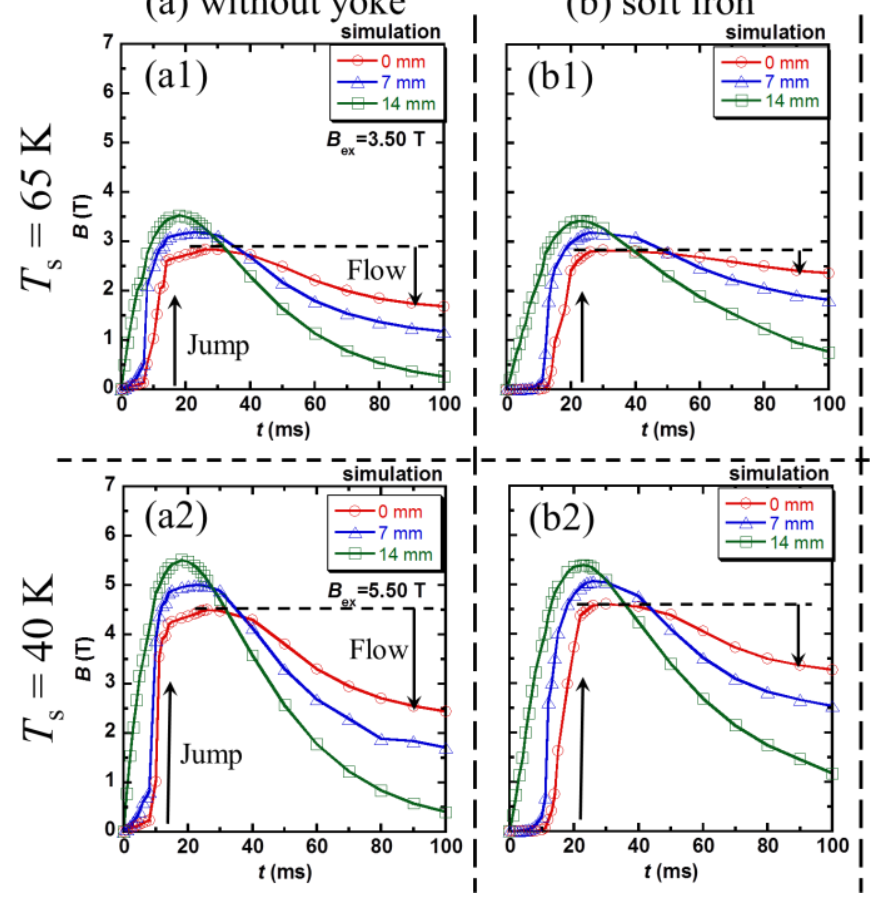

(b) soft iron
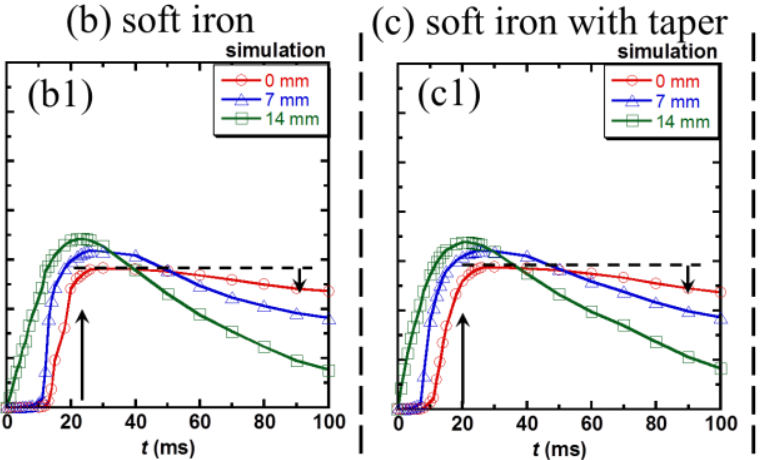

(d) permendur

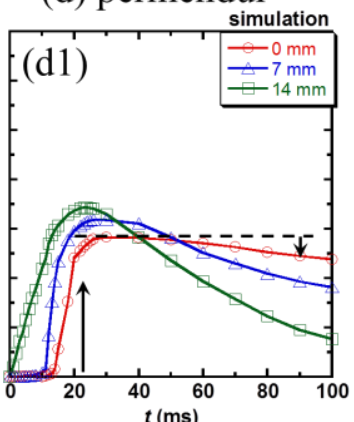

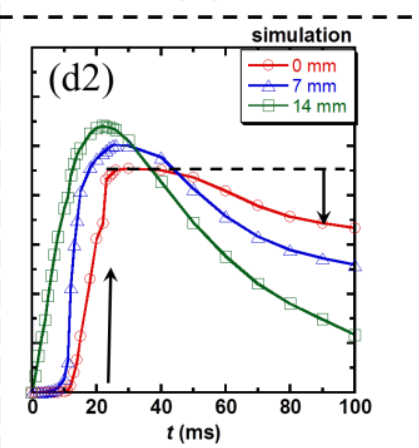

Fig. 15. Time dependence of the local field on the top surface of the bulk at each position $r=0 \mathrm{~mm}$ (center), $7 \mathrm{~mm}$ (middle) and $14 \mathrm{~mm}$ (edge) from the bulk center, after applying $B_{\mathrm{ex}}=3.50 \mathrm{~T}$ at $65 \mathrm{~K}$ (upper panels) using the split coil (a1) without any yoke and with the three types of yoke, (b1) soft iron, (c1) tapered soft iron and (d1) permendur, and after applying $B_{\text {ex }}=5.50 \mathrm{~T}$ at 40 $\mathrm{K}$ (lower panels), using the split coil (a2) without any yoke and with the three types of yoke, (b2) soft iron, (c2) tapered soft iron and (d2) permendur.

Figure 16 shows the time dependence of the flux increase rate, $R_{\mathrm{F}}$, after applying the pulsed field, $B_{\mathrm{ex}}=5.5 \mathrm{~T}$ at $40 \mathrm{~K}$. These figures, which were calculated from Figs. 15(a2) (d2), show similar plots to those shown in Fig. 7. The maximum $R_{\mathrm{F}}$ value for any case with a yoke was fairly smaller than without any yoke because the magnetic flux gradually intruded in several stages with small flux jumps, showing that the yoke acted to suppress the dynamical motion of the magnetic flux. This positive effect of the yoke resulted from its electrical conductivity, $\sigma$, which acted to delay the 
flux movement in the bulk by induced eddy currents, flowing within the yoke.

Figure 17 shows the maximum temperature rise, $\Delta T$, estimated on the edge of the bulk, as a function of the applied field, $B_{\text {ex }}$, for the split coil at $65 \mathrm{~K}$ and $40 \mathrm{~K}$. The magnitude of $\Delta T$ was decreased by the insertion of iron yoke, which was the same manner of the magnitude of $R_{\mathrm{F}}$ shown in Fig. 17. The reduction of $\Delta T$ was relatively small, compared to the experimental results in Fig. 8, because the small $B_{0}$ value of 0.04 in a Kim model, which seriously suppresses the $J_{\mathrm{c}}$ value under the magnetic field, reduces the temperature rise, even though the $\alpha$ value is higher than the present bulk in Eq. (1).

The effect of the yoke insertion on the PFM results can be related to the electrical properties of the bulk superconductor, and the saturation magnetic flux density, $B_{\text {sat }}$, and electrical conductivity, $\sigma$, of the yoke material. $B_{\text {sat }}$ acts to reduce the flux flow in the descending stage, and $\sigma$ acts to suppress $R_{\mathrm{F}}$ and reduces the heat generated in the bulk, because of eddy currents flowing in the yoke that opposes the magnetization. Hence, a yoke with higher $B_{\text {sat }}$ and $\sigma$ is desirable to improve the final trapped field by PFM from both electromagnetic and thermal points of view.

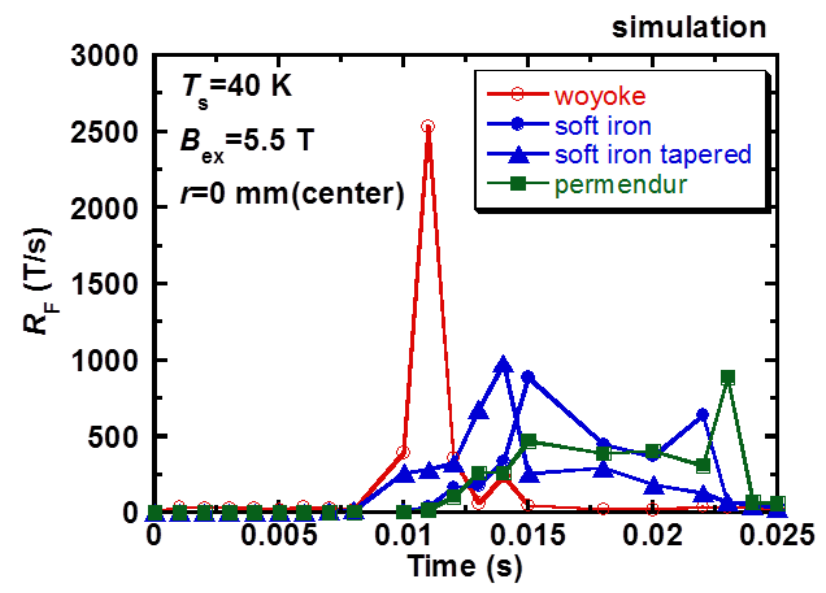

Fig. 16. Time dependence of the flux increase rate, $R_{\mathrm{F}}$, at the center of the bulk surface after applying $B_{\text {ex }}=5.5 \mathrm{~T}$ at $40 \mathrm{~K}$, for all cases with and without the three types of yoke. $R_{\mathrm{F}}$ was calculated from $\mathrm{d} B_{\mathrm{y}}(t) / \mathrm{d} t$ in Figs. 15(a2) (d2).

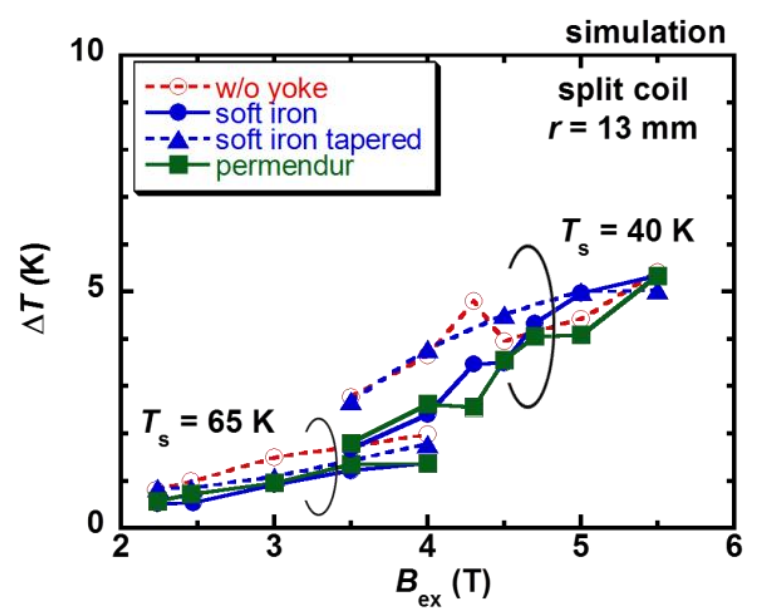


Fig. 17. Maximum temperature rise, $\Delta T$, extracted from the edge of the bulk, as a function of the applied field, $B_{\mathrm{ex}}$, for the split coil at $65 \mathrm{~K}$ and $40 \mathrm{~K}$.

\section{Summary}

We have investigated both experimentally and numerically the trapped field characteristics of a standard Y-Ba-Cu-O bulk magnetized by PFM using a split coil with three kinds of iron yokes inserted in the bores of coil. The important results and conclusions obtained from this study are summarized as follows.

(1) A higher saturation magnetic flux density, $B_{\text {sat }}$, of the yoke material was effective to reduce flux flow in the descending stage of the pulsed field. A maximum trapped field of $2.93 \mathrm{~T}$ at 40 $\mathrm{K}$ was achieved on the center of the bulk surface using the split coil with the permendur yoke with a flat surface. The difference in the trapped field between the soft iron and permendur is reasonably small because the $B_{\text {sat }}$ value is not significantly different.

(2) A higher and conical trapped field can be achieved in the case of soft iron with a flat surface. In the case of soft iron with a taper, the trapped field was expected to be enhanced because of the concentration of the magnetic flux. However, the maximum trapped field was lower than that for a flat surface. The magnetic flux cannot concentrate at the center of the coil because the applied field is higher than $B_{\text {sat }}$ and thus the tapered shape is ineffective in this respect.

(3) The results of the numerical simulation qualitatively reproduced the experimental results, such as the applied field dependence of the trapped field. The typical behavior of the magnetic flux, such as a flux jump, can be also reproduced by an assumption of $J_{\mathrm{c}}(B)$ characteristics based on a Kim model and a higher $J_{\mathrm{c}}$ value under zero magnetic field, compared to the measured one.

(4) The effect of the yoke insertion was also simulated using a realistic saturation magnetic flux density, $B_{\text {sat }}$, and electrical conductivity, $\sigma$, of the yoke materials. The contribution of the yoke on the PFM results is more influential during the descending stage of the pulse. $B_{\text {sat }}$ of the yoke material acts to reduce flux flow, such that the magnetic flux still remains trapped with minimal flux flow, even after the whole process of magnetization. A large $\sigma$ value of the yoke material also acts to reduce the flux increase rate, $R_{\mathrm{F}}$, of the flux intruding the bulk because of eddy currents that flow in the yoke that oppose the magnetization. This reduces the temperature rise, $\Delta T$, in the bulk, which improves the final trapped field.

\section{Acknowledgements}

This research is partially supported by JSPS KAKENHI grant number 15K04646. Dr. Mark Ainslie would like to acknowledge the support of a Royal Academy of Engineering Research Fellowship. 


\section{References}

[1] D. A. Cardwell, Mater. Sci. Eng. B53 (1998) 1-10.

[2] S. Nariki, N. Sakai, M. Murakami, Supercond. Sci. Technol. 18 (2005) S126-30.

[3] H. Hayashi, K. Tsutsumi, N. Saho, N. Nishizima, K. Asano, Physica C. 745 (2003) 392-396.

[4] F. Mishima, S. Takeda, Y. Izumi, S. Nishijima, IEEE Trans. Appl. Supercond. 17 (2007) 2303.

[5] Y. Yanagi, T. Matsuda, H. Hazama, K. Yokouchi, M. Yoshikawa, Y. Itoh, T. Oka, H. Ikuta, U. Mizutani, Physica C. 764 (2005) 426-431.

[6] J. H. Durrell, A. R. Dennis, J. Jaroszynski, M. D. Ainslie, K. G. B. Palmer, Y-H. Shi, A. M. Campbell, H. Hull, M. Strasik, E. E. Hellstrom, D. A. Cardwell, Supercond. Sci. Technol. 27 (2014) 082001.

[7] M. D. Ainslie, H. Fujishiro, T. Ujiie, J. Zou, A. R. Dennis, Y-H. Shi, D. A. Cardwell, Supercond. Sci. Technol. 27 (2014) 065008.

[8] H. Fujishiro, T. Oka, K. Yokoyama, K. Noto, Supercond. Sci. Technol. 16 (2003) 809.

[9] H. Fujishiro, K. Yokoyama, T. Oka, K. Noto, Supercond. Sci. Technol. 17 (2004) 51.

[10] H. Fujishiro, T. Oka, K. Yokoyama, M. Kaneyama, K. Noto, IEEE Trans. Appl. Supercond. 14 (2004) 1054.

[11] H. Fujishiro, T. Tateiwa, A. Fujiwara, T. Oka, H. Hayashi, Physica C. 445-448 (2006) 334-338.

[12] H. Ikuta, H. Ishihara, Y. Yanagi, Y. Itoh, U. Mizutani, Supercond. Sci. Technol. 15 (2002) 606.

[13] M. P. Philippe, M. D. Ainslie, L. Wera, J-F Fagnard, A. R. Dennis, Y-H. Shi, B. Vanderheyden, P. Vanderbemden, Supercond. Sci. Technol. 28 (2015) 095008.

[14] M. D. Ainslie, H. Fujishiro, H. Mochiuki, K. Takahashi, Y-H. Shi, D. K. Nambri, J. Zou, D. Zhou, A. R. Dennis, D. A. Cardwell, Supercond. Sci. Technol. 29 (2016) 074003.

[15] K. Takahashi, M. D. Ainslie, H. Fujishiro, T. Naito, IEEE Trans. Appl. Supercond. 27 (2017) 6800205.

[16] H. Fujishiro, H. Mochizuki, M. D. Ainslie, T. Naito, Supercond. Sci. Technol. 29 (2016) 084001.

[17] M. D. Ainslie, H. Fujishiro, Supercond. Sci. Technol. 28 (2015) 053002.

[18] M. D. Ainslie, J. Zou, H. Mochizuki, H. Fujishiro, Y-H. Shi, A. R. Dennis, D. A. Cardwell, Supercond. Sci. Technol. 28 (2015) 125002.

[19] S. Zou, V. M. R. Zermeno, F. Grilli, IEEE Trans. Appl. Supercond. 26 (2016) 4702405.

[20] Z. Xu, R. Lewin, A. M. Campbell, D. A. Cardwell, Supercond. Sci. Technol. 25 (2012) 025016.

[21] K. Berger, J. Leveque, D. Netter, B. Douine, A. Rezzoug, IEEE Trans. Appl. Supercond. 17 (2007) 3028-3031.

[22] Z. Xu, R. Lewin, A. M. Campbell, D. A. Cardwell, H. Jones, J. Phys. Conf. Series. 234 (2010) 012049.

[23] M. Tsuchimoto, H. Waki, Y. Itoh, Y. Yanagi, T. Oka, Y. Yamada, U. Mizutani, Cryogenics. 37 
(1997) 43-47.

[24] Y. Shi, N. H. Babu, D. A. Cardwell, Supercond. Sci. Technol. 18 (2005) L13-6.

[25] Y. Yanagi, Y. Itoh, M. Yoshikawa, T. Oka, H. Ikuta, U. Mizutani, Supercond. Sci. Technol. 18 (2005) 839-849.

[26] R. Weinstein, D. Parks, R-P. Sawh, K. Carpenter, K. Davey, J. Appl. Phys. 119 (2016) 133906.

[27] H. Fujishiro, T. Naito, M. Oyama, Supercond. Sci. Technol. 24 (2011) 075015.

[28] T. Oka, K. Hara, J. Ogawa, S. Fukui, T. Sato, K. Yokoyama, A. Murakami, M. Langer, IEEE Trans. Appl. Supercond. 26 (2016) 1-4.

[29] U. Mizutani, T. Oka, Y. Itoh, Y. Yanagi, M. Yoshikawa, H. Ikuta, Appl. Supercond. 6 (1998) 235.

[30] H. Ohsaki, T. Shimosaki, N. Nozawa, Supercond. Sci. Technol. 15 (2002) 754-758.

[31] Y. Komi, M. Sekino, H. Ohsaki, Physica C. 469 (2009) 1262-1265.

[32] H. Fujishiro, T. Naito, Supercond. Sci. Technol. 23 (2010) 105021.

[33] J. Duron, F. Grilli, B. Dutoit, S. Stavrev, Physica C. 401 (2004) 231-235.

[34] J. Xia, Y. Zhou, Cryogenics. 69 (2015) 1-9.

[35] M. D. Ainslie, D. Zhou, H. Fujishiro, K. Takahashi, Y-H. Shi, J. H. Durrell, Supercond. Sci. Technol. 29 (2016) 124004. 